\title{
O "gibi" como instrumento à promoção da saúde e prevenção do abuso de drogas: relato de experiência de residentes multiprofissionais em Saúde da Família
}

The "comic book" as an instrument for health promotion and drug abuse prevention: report on the experience of multiprofessional residents in Family Health

\author{
El "cómics" como instrumento a la promoción de la salud y prevención del abuso de \\ drogas: relato de experiencia de residentes multiprofesionales en Salud de la Familia
}

Fernanda Ramos da Silva ${ }^{*}$, Eduardo Padilha Barros ${ }^{1}$, Márcio Vinícius de Gouveia Affonso ${ }^{1}$, Alace da Silva Peres ${ }^{1}$, Michelle Suany Ferreira Monteiro ${ }^{1}$, Alessandra dos Santos Tavares Vieira ${ }^{1}$, Pettra Blanco Lira Matos ${ }^{1}$, Yasmin Caroline Gomes Maia'1, Lucas Oliveira da Silva', Bianca Caldas da Silva Mendes ${ }^{1}$, Camila Rodrigues Veloso', Sâmia Cristine Rabelo Borges'1.

\section{RESUMO}

Objetivo: Relatar a experiência de residentes multiprofissionais em Saúde da Família sobre uma atividade educativa e preventiva à respeito do uso de álcool, tabaco e outras drogas, utilizando o gibi educativo, em uma escola municipal da periferia de Belém-PA. Relato de experiência: A residência multiprofissional em Saúde da Família atua de maneira intersetorial. Um dos cenários de atuação, é o ambiente escolar, por meio do Programa Saúde na Escola (PSE). Dessa forma, os residentes realizaram ação de promoção da saúde com 5 (cinco) turmas do "Fundamental menor" ( $1^{\circ}$ ao $5^{\circ}$ ano do primeiro ciclo escolar) de uma escola municipal da rede pública de Belém-PA sobre a prevenção do abuso de álcool, tabaco e outras drogas. Para abordar a temática, utilizou-se uma metodologia lúdica e interativa por intermédio da leitura e discussão de um gibi educativo do cartunista Maurício de Sousa, intitulado "Turma da Mônica em: Uma história que precisa ter fim". Conclusão: A utilização do gibi educativo como um instrumento lúdico, naquele cenário, ampliou o universo de possibilidades para alcançar o público-alvo de forma eficaz. Aliado a isso, a utilização da metodologia proposta contribuindo positivamente para a implementação do processo de trabalho e a qualidade na formação dos residentes em Saúde da Família, fortalecendo e facilitando atuação intersetorial para a promoção e prevenção da saúde.

Palavras-chave: Histórias em Quadrinhos, Promoção da Saúde, Atenção Primária à Saúde.

\begin{abstract}
Objective: To report the experience of multiprofessional residents in Family Health on an educational and preventive activity regarding the use of alcohol, tobacco and other drugs, using the educational comic book, in a municipal school in the periphery of Belém-PA. Experience report: The multiprofessional residence in Family Health operates in an intersectoral way. One of the action scenarios is the school environment, through the Health in School Program (HSP). Thus, residents carried out a health promotion action with 5 (five) classes of "Fundamental Minor" (1st to 5th year of the first school year) of a municipal school of the public network of Belém-PA on the prevention of alcohol abuse, tobacco and other drugs. To approach the theme, a playful and interactive methodology was used through the reading and discussion of an educational comic book by the cartoonist Maurício de Sousa, entitled "Turma da Mônicaem: Uma história que precisaterfim" Conclusion: The use of the educational comic as a playful instrument, in that scenario, extended the universe of possibilities to reach the target audience in an effective way. Allied to this, the use of the proposed methodology contributing
\end{abstract}

1Universidade do Estado do Pará (UEPA), Belém-PA. *E-mal: nanda_ramos@live.com 
positively to the implementation of the work process and the quality in the training of the residents in Family Health, strengthening and facilitating intersectorial action for the promotion and prevention of health.

Key words: Comics, Health promotion, Primary Health Care.

\section{RESUMEN}

Objetivo: Informar la experiencia de residentes multiprofesionales en Salud de la Familia sobre una actividad educativa y preventiva al respecto del uso de alcohol, tabaco y otras drogas, utilizando el gibi educativo, en una escuela municipal de la periferia de Belém-PA. Relato de experiencia: La residencia multiprofesional en Salud de la Familia actúa de manera intersectorial. Uno de los escenarios de actuación, es el ambiente escolar, a través del Programa Salud en la Escuela (PSE). De esta forma, los residentes realizaron acción de promoción de la salud con 5 (cinco) grupos del "Fundamental menor" ( $1^{\circ}$ al $5^{\circ}$ año del primer ciclo escolar) de una escuela municipal de la red pública de Belém-PA sobre la prevención del abuso de alcohol, tabaco y otras drogas. Para abordar la temática, se utilizó una metodología lúdica e interactiva por medio de la lectura y discusión de un cómic educativo del caricaturista Mauricio de Sousa, titulado "Turma da Mônica em: Uma história que precisa ter fim". Conclusión: La utilización del gibi educativo como un instrumento lúdico, en aquel escenario, amplió el universo de posibilidades para alcanzar el público objetivo de forma eficaz. Aliado a ello, la utilización de la metodología propuesta contribuyendo positivamente a la implementación del proceso de trabajo y la calidad en la formación de los residentes en Salud de la Familia, fortaleciendo y facilitando actuación intersectorial para la promoción y prevención de la salud.

Palabras clave: Cómics, Promoción de la salud, Atención Primaria a la Salud.

\section{INTRODUÇÃO}

O consumo de álcool, tabaco e outras drogas é um fenômeno mundial complexo, multicausal e é visto como um grave problema de saúde pública, que afeta crianças e adolescentes (HENRIQUES BD et al., 2016). De acordo com a United Nations Office for DrugControl and Crime Prevention - UNODC (2017), em 2015, cerca de 250 milhões de pessoas utilizavam drogas. O consumo de drogas na sociedade moderna está fortemente atrelado à condição de vulnerabilidade e ao nível socioeconômico da população (RUOTTI C, MASSA VC, PERES MFT, 2011).

Estrada e Pilipiak (2016) destacam que o uso de álcool e demais drogas está associado a uma série de comportamentos de risco, aumentando a chance de envolvimento em acidentes, violência sexual e participação em grupos violentos, fortemente associados à morte violenta, queda no desempenho escolar, dificuldades de aprendizado, prejuízo no desenvolvimento e estruturação das habilidades cognitivocomportamentais e emocionais da pessoa. Por sua gravidade e abrangência, é necessário que as ações de enfrentamento se deem além do campo da saúde.

Cavalcante (2014) destaca que a criação de metodologias educacionais para a educação em saúde possibilita aos profissionais dessa área adaptar sua atuação no contexto educacional a cada segmento social, respeitando suas crenças e limitações, com estratégias palpáveis, de fácil linguagem e interpretação e com atrativos visuais. Portanto, à articulação Intersetorial é indispensável, devem ser adotadas estratégias que possam enfrentar de fatoas situações de violência urbana, e redução das iniquidades sociais históricas, para garantir o acesso à educação, ao trabalho, ao lazer e a cultura (HENRIQUES BD et al., 2016).

Nesse contexto, é inserido o Programa Saúde na Escola, instituído em 2007, pelo Decreto Presidencial no 6.286, que se trata de uma política pública com articulação dos setores saúde e educação, com ampla participação da comunidade escolar. De forma continuada, promove a incorporação da cultura da prevenção de doenças, violências e outros agravos, desse modo privilegiando a escola como um espaço para articulação das políticas voltadas para as crianças, adolescentes, jovens e adultos, mediante participação como sujeitos desse processo (MENDONÇA FILHO J, 2017). 
Nesse cenário, é imperativo fazer uso de diversas estratégias para atingir de forma positiva as crianças, por meio da educação em saúde. As Histórias em Quadrinhos (HQs) fazem parte do universo infantojuvenil e sua evolução como mídia de entretenimento demonstra novas possibilidades de uso e demonstra ter influência sobre esse público (SOUZA RQ , 2017). A comunicação visual é também influente; uma boa ilustração, além de transmitir uma ideia e vender um conceito, é capaz de permanecer na memória do públicoalvo por um longo tempo (ARAÚJO ACC, 2017).

Diante disso, o estudo busca relatar a experiência de residentes multiprofissionais em Saúde da Família, da Universidade do Estado do Pará (UEPA) na realização de uma atividade educativa e preventiva a respeito do abuso de álcool, tabaco e outras drogas, utilizando o "gibi educativo", em uma escola municipal da periferia de Belém-PA, no ano de 2018.

\section{MÉTODOS}

Trata-se de um estudo descritivo, do tipo relato de experiência, que apresenta a vivência de residentes multiprofissionais (cirurgiões-dentistas, enfermeiros, fisioterapeutas e terapeutas ocupacionais), sobre uma ação de promoção da saúde, voltada à prevenção do abuso de drogas lícitas e ilícitas.

\section{RELATO DE EXPERIÊNCIA}

A residência multiprofissional em Saúde da Família atua de maneira intersetorial, realizando ações em saúde para além dos muros do ambiente da Unidade Básica de Saúde (UBS). Um dos cenários de atuação, dentre vários, é o ambiente escolar, por meio do Programa Saúde na Escola (PSE). Dessa forma, os residentes realizaram ação em saúde com 5 (cinco) turmas do "Fundamental menor" ( $1^{\circ}$ ao $5^{\circ}$ ano do primeiro ciclo escolar) de uma escola municipal da rede pública de Belém-PA sobre a prevenção do abuso de álcool, tabaco e outras drogas.

Para abordar a temática, utilizou-se uma metodologia lúdica e interativa por intermédio da leitura e discussão de um gibi educativo do cartunista Maurício de Sousa, intitulado "Turma da Mônica em: Uma história que precisa ter fim", o qual tem acesso livre e permitido pela Secretaria Nacional Antidrogas e pelo Governo Federal. Essa revista em quadrinhos aborda o tema de forma bem acessível à compreensão de crianças, facilitando o processo interativo. As turmas participaram uma por vez; o gibi foi exposto para todas as turmas por meio do uso de datashow, em que as imagens da história em quadrinhos eram projetadas no quadro branco e todos os escolares acompanhavam a leitura e discussão do tema simultaneamente.

É de extrema importância relatar que o planejamento das atividades foi realizado em comunhão com os setores educação, representado pela direção e coordenação da escola; o setor saúde, representado pela secretaria municipal de saúde; e os residentes, tendo em vista a proposta de ensino e serviço desse programa de pós-graduação.

Dessa forma, fica exposto que a efetividade das ações promotoras de saúde necessita da interação entre os setores envolvidos, em que cada um irá propor a melhor forma de trabalho e as suas deficiências, com o intuito de eliminá-las ou reduzi-las ao máximo.

\section{DISCUSSÃo}

O conceito moderno de promoção da saúde, bem como sua prática, emerge e se desenvolve mais fortemente nos últimos 20 anos, sobremaneira no Canadá, nos Estados Unidos e em países da Europa Ocidental (CZERESNIA D e FREITAS CM, 2003). A promoção da saúde propõe cinco campos principais de ação, surgidos a partir da Carta de Ottawa: elaboração e implementação de políticas públicas saudáveis; criação de ambientes favoráveis à saúde; reforço da ação comunitária; desenvolvimento de habilidades pessoais e reorientação do sistema de saúde. 
É certo que estamos distantes de atingir integralmente todos os campos, no entanto, temos avançado, a exemplo da publicação da Política Nacional de Promoção da Saúde (PNPS) em 2006. A PNPS contém em si o objetivo geral de promover a qualidade de vida e reduzir vulnerabilidades e riscos à saúde relacionados aos seus determinantes e condicionantes (BRASIL, 2006).

A PNPS afirma que a escola é um espaço de relações, portanto, deve ser valorizada como espaço para o desenvolvimento crítico e político, espaço portanto, de promoção da saúde. Partindo dessa premissa em 2007 o Ministério da Saúde instituiu o Programa Saúde na Escola (PSE) tendo entre seus objetivos principais a promoção da cultura de paz no ambiente escolar e o enfrentamento das vulnerabilidades, no campo da saúde, que possam comprometer o pleno desenvolvimento escolar (BRASIL, 2009).

A promoção da cultura de paz foi o objetivo que norteou a atividade aqui relatada. Participaram das atividades referentes à prevenção de abuso de álcool, tabaco e outras drogas 111 escolares, com idade entre 7 e 11 anos. A partir da utilização do instrumento foi possível trabalhar, de forma lúdica e interativa, assuntos que são realidade do cenário social e constituintes desse, tais como: os males causados à saúde pelo uso de drogas (lícitas e ilícitas); a noção de criminalidade referente ao comércio de drogas ilícitas; a comunicação à adultos responsáveis/familiares, professores, diretores das escolas - em casos de uso de drogas por amigos e/ou conhecidos, a fim de alertar sobre o risco; e o tratamento das pessoas que se tornam usuárias dependentes das mesmas (SOUZA RQ et al., 2015).

A presença de educadores durante a atividade realizada também favoreceu o entendimento por parte dos alunos, uma vez que o vínculo criado entre esses atores fez com que a figura dos residentes fosse respeitada e compreendida. Assim, os alunos permaneceram atentos à atividade, sem interrupções indevidas, participando com perguntas e questionamentos relacionados ao assunto abordado.

Os escolares se mostraram muito receptivos e inclinados à participação pelo tipo de abordagem adotado pelos residentes e pela utilização da revista em quadrinhos. Um estudo de Prado, Sousa Júnior e Pires (2017) demonstrou, através de pesquisa descritiva documental entre os anos de 2000 a 2016, que as Histórias em Quadrinhos podem ser um instrumento pedagógico e informativo para a educação e promoção da saúde por atingirem uma maior clientela, "e são recomendadas para a difusão de conhecimentos para mudança de hábitos nocivos e insalubres, objetivando melhor qualidade de vida em caráter desenvolvimentista e sustentável".

Por se tratar de uma metodologia ativa, a contribuição dos mesmos superou as expectativas idealizadas pelos facilitadores da atividade, pois estes demonstraram ter conhecimento prévio sobre drogas, tanto lícitas quanto ilícitas, dando opiniões sobre o assunto, alguns relataram experiências vivenciadas em seus meios de convívio, de forma indireta por meio da mídia televisa, por momentos promovidos pelos professores em sala de aula e de maneira mais próxima, por meio de relatos de experiência no meio familiar, através de familiares e parentes.

A infância é uma fase promissora para a edificação de costumes e atitudes, a escola acaba assumindo papel importante nesse processo, onde oambiente escolar é um local que oferece múltiplas possibilidades de educar por meio da construção de conhecimentos resultantes do confronto dos diferentes saberes - em que todos os atores sociais envolvidos, tornam-se potenciais agentes multiplicadores de promoção da saúde (GUIMARÃES MN et al., 2014).

Dentro desse contexto, Graciano et al. (2015, p.34) ressaltam que a "promoção da saúde nas escolas envolve a criação de entornos saudáveis, a educação em saúde integral e a provisão de serviços de saúde", com comunicação por meio de esforços e recursos multissetoriais, contribuindo para o enfrentamento de situações de vulnerabilidade.

As ações em saúde realizadas nesse cenário favorecem aos beneficiados ampliação de seus conhecimentos sobre diferentes temas abordados, permitindo a estes conhecer e lidar com fatores de risco e fortalecer fatores protetivos, além de apresentar impacto direto na promoção e proteção à saúde, 
influenciando também de maneira positiva em sua autonomia (GRACIANO AMC et al., 2015). Ao viabilizar a garantia de qualidade de vida, promove-se saúde.

Neste processo, a participação de atores de diferentes setores é essencial, característica essa que está presente na atividade relatada neste trabalho e se tornou um diferencial com impactos positivos nos resultados obtidos. Ademais, o conhecimento adquirido pelos escolares poderá influenciar não somente nas decisões tomadas por estes durante sua vida e amadurecimento, mas também poderão ser compartilhadas e servirão de espelho para outrem. Portanto, poderão atuar como agentes multiplicadores de saúde, contribuindo para o bem estar comunitário.

\section{CONSIDERAÇÕES FINAIS}

A utilização do gibi educativo como um instrumento lúdico para crianças de 7 a 11 anos foi considerada, naquele cenário, como de ótima aceitação, tanto pelos educandos, quanto pela coordenação e direção da escola, por se tratar de uma linguagem fácil, ilustrativa e sem imagens ou situações consideradas inapropriadas. Aliado a isso, a participação dos residentes de diferentes categorias profissionais permitiu além da atuação interprofissional, a troca de saberes, em que todos os envolvidos se tornaram aptos a atuarem como agentes multiplicadores de promoção da saúde. Aliado a isso, a utilização da metodologia proposta ampliou o universo de possibilidades para alcançar o público-alvo de forma eficaz, contribuindo positivamente para a implementação do processo de trabalho e a qualidade na formação dos residentes em Saúde da Família, fortalecendo e facilitando atuação intersetorial para a promoção e prevenção da saúde.

\section{REFERÊNCIAS}

1. ARAÚJO ACC. Comunicação visual no processo de geração de ideias: uma proposta para a técnica de criatividade Creation. Dissertação (mestrado) - Universidade Federal do Rio Grande do Norte, Centro de Tecnologia, Pós-graduação em Engenharia de Produção, Natal, 2017. 149f. Disponível em: https://repositorio.ufrn.br/jspui/bitstream/123456789/25967/1/Comunica\%C3\%A7\%C3\%A3ovisualprocesso Ara \%C3\%BAjo 2018.pdf. Acesso em: 21 mar. 2018.

2. BRASIL. Ministério da Saúde. Secretaria de Vigilancia em Saúde. Política Nacional de Promoçao da Saúde: Portaria n 68 MS/GM, de 30/3/2006. 60p. (Série B. Textos Básicos em Saúde).

3. Ministério da Saúde. Secretaria de Atenção à Saúde. Departamento de Atenção Básica. Saúde na escola. Brasília: Ministério da Saúde. 2009.

4. CAVALCANTE AL. Aplicação de tecnologias educacionais na educação em saúde. Portal da educação, 2014. Disponível em: www.portaleducacao.com.br/conteudo/artigos/idiomas/aplicacao-de-tecnologias-educacionaisna-educacao-em-saude/58400. Acesso em: 3 mar. 2018.

5. CZERESNIA D, FREITAS CM de. (org.). Promoção da saúde: conceitos, reflexões, tendência. Rio de Janeiro: editora Fiocruz, 2003.

6. ESTRADA AA, FILIPIAK D. O consumo de álcool na adolescência: reflexões para além do ambiente escolar. Cadernos PDE. Os desafios da escola pública paranaense na perspectiva do professor PDE. Versão online. ISBN: 978-85-8015-093-3. 2016.

7. HENRIQUES BD et al. O uso de crack e outras drogas por crianças e adolescentes e suas repercussões no ambiente familiar. Escola Anna Nery, 20(4) Out-Dez 2016.

8. GRACIANO AMC, et al. Promoção da Saúde na Escola: história e perspectivas. J. Health Biol Sci. 2015; 3(1):3438. Disponível em: http://periodicos.unichristus.edu.br/index.php/jhbs/article/view/110/100. Acesso em: 12 jan 2019.

9. GUIMARÃES MN et al. Escola: espaço de construção do conhecimento. VI FIPED - Fórum Internacional de Pedagogia. Santa Maria - Rio Grande do Sul, jul-ago 2014. Disponível em: http://editorarealize.com.br/revistas/fiped/trabalhos/Modalidade 2datahora 25052014180513 idinscrito 1225 4fc6da7bf11dada67f42200495a3dd64.pdf. Acesso em: 12 jan 2019. 
10. HENRIQUES BD, ROCHA RL, REINALDO AMS. Uso de Crack e outras drogas entre crianças e adolescentes e seu impacto no ambiente familiar: Uma revisão integrativa da literatura. Texto Contexto Enferm, 2016; 25(3):e1100015.

11. MENDONÇA FILHO J. Ministérios da Saúde e Educação publicam nova portaria do Programa Saúde na Escola. Governo do Estado de Santa Catarina. 2017. Disponível em: http://portalses.saude.sc.gov.br/index.php?option=com content\&view=article\&id=2584\&ltemid=484. Acessoem: 15 mai. 2018.

12. PRADO CC, SOUSA JÚNIOR CE, PIRES ML. Histórias em quadrinhos: uma ferramenta para a educação e promoção da saúde. Reciis - Rev Eletron Comun Inf Inov Saúde, abr.-jun.; 11(2). 2017. p. 1. Disponível em: https://www.reciis.icict.fiocruz.br/index.php/reciis/article/view/1238. Acesso em: 10 Mai. 2018.

13. RUOTTI C, MASSA VC, PERES MFT. Vulnerabilidade e violência: uma nova concepção de risco para o estudo dos homicídios de jovens. Interface - Comunic., Saude, Educ., v.15, n.37, p.377-89, abr./jun. 2011. Disponível em: http://www.scielo.br/pdf/icse/v15n37/aop0111. Acesso em: 5 jun 2018.

14. SOUSA MA Turma da Mônica em: Uma história que precisa ter fim. 2011. Disponível em: http://www.sbp.com.br/fileadmin/user upload/Revista Da Turma Da Monica Sobre Drogas.pdf. Acesso em: 19 abr 2018.

15. SOUZA MR et al. Juventude e drogas: uma intervenção sob a perspectiva da Psicologia Social. Pesquisas e Práticas Psicossociais, 10(1), São João del-Rei, janeiro/junho 2015.

16. SOUZA RQ. Histórias em quadrinhos como fonte de informação em incentivo à leitura. Trabalho de Conclusão de Curso (graduação) - Universidade Federal do Pará, Instituto de Ciências Sociais Aplicadas, Faculdade de Biblioteconomia, $2017 . \quad 54 f . \quad$ Disponível em: http://bdm.ufpa.br/jspui/bitstream/prefix/166/1/TCC HistoriasQuadrinhosFonte.pdf. Acesso em: 13 jun 2018.

17. ORGANIZAÇÃO DAS NAÇÕES UNIDAS. 2017. Disponível em: http://www.unodc.org/lpobrazil/pt/frontpage/2017/06/cerca-de-29-5-milhes-de-pessoas-em-todo-o-mundo-sofrem-de-transtornosprovocados-pelo-uso-de-drogas--os-opiides-so-os-mais-prejudiciais -aponta-relatrio-mundial-sobre-drogas2017-do-unodc.html. Acesso em: 08 mai. 2018. 